\section{Perfil dos indicadores de gordura e massa muscular corporal dos idosos de Fortaleza, Ceará, Brasil}

\author{
Trends in body fat and muscle mass among elderly \\ individuals in Fortaleza, Ceará State, Brazil
}

\begin{abstract}
The aim of this study was to describe trends in body fat and muscle mass among elderly individuals in Fortaleza, Ceará State, Brazil. This was a population-based, cross-sectional, household survey of elderly individuals $(\geq 60$ years) ( $n=483)$ in Fortaleza. The variables analyzed were: triceps skinfold thickness (TST), upper arm fat area (UFA), mid upper arm circumference (MAC), arm muscle circumference (AMC), arm muscle area (AMA), and corrected arm muscle area (CAMA). Mean TST and UFA were significantly higher in women than men ( $p<$ 0.05). Mean AMC, AMA, and CAMA were higher in men than women $(p<0.05)$. For all the variables, the mean and 50th percentile decreased with age, except the TST percentile for women. Anthropometric and body composition patterns in the elderly in this study showed similar trends to those of other studies, but with different values. The values could thus be useful as references for the elderly in Fortaleza.
\end{abstract}

Anthropometry; Body Composition; Elderly
Tarciana Nobre de Menezes 1

Maria de Fátima Nunes Marucci 2

\section{Introdução}

Com o envelhecimento ocorrem alterações corporais que podem interferir no estado nutricional do idoso. A massa muscular tende a diminuir, assim como há modificações no padrão de distribuição da gordura corporal, onde o tecido gorduroso dos braços e pernas diminui, no entanto aumenta no tronco 1,2 . Por conta disso, ocorrem modificações em alguns indicadores de gordura e de massa muscular, os quais são comumente avaliados em estudos antropométricos com idosos. São eles: dobra cutânea tricipital (DCT), área de gordura do braço (AGB), circunferência muscular do braço (CMB), área muscular do braço (AMB) e área muscular do braço corrigida (AMBc) 3,4,5,6,7,8,9,10,11,12,13.

Diante dessas alterações, é necessário que haja avaliação das mesmas, no sentido de identificar se são comuns do envelhecimento ou resultantes de doenças que acometem o idoso. No entanto, para utilizar essas variáveis como forma de avaliar o estado nutricional de idosos é necessário que haja padrões antropométricos de referência, com os quais as medidas de cada indivíduo sejam comparadas. Estudos antropométricos têm sido realizados com idosos em diversos países, como forma de descrever o perfil antropométrico e estabelecer padrões de referência locais que auxiliem na avaliação nutricional desses idosos 4,6,7,8,9,11,12,13, no entanto, 
ainda são poucos os países que possuem estudos populacionais nacionais 3,9 .

Nos Estados Unidos, estudo com dados referentes à terceira National Health and Nutrition Examination Survey (NHANES III) apresenta valores de referência antropométricos de uma amostra nacionalmente representativa dos idosos 9. Anteriormente, Frisancho ${ }^{3}$, a partir dos dados da primeira Health and Nutrition Examination Survey (HANES I), apresenta a distribuição percentual de algumas variáveis antropométricas dos idosos participantes do estudo.

Estudos antropométricos populacionais realizados com idosos no Brasil ainda são poucos 12,14,15. Desses estudos, apenas o de Barbosa et al. 12, que foi realizado com uma amostra representativa dos idosos não institucionalizados de São Paulo, apresenta distribuição em percentil das variáveis antropométricas. Menezes \& Marucci 13 , em estudo com idosos residentes em instituições geriátricas da cidade de Fortaleza, Ceará, também apresentam as informações em percentil e ainda sugerem que os valores sejam utilizados como forma de auxiliar na avaliação nutricional de idosos institucionalizados. Essa forma de apresentação dos dados é considerada a mais sensível que as variáveis antropométricas podem ser apresentadas, se comparadas à apresentação por meio das médias 16 .

A inexistência de padrões de referência antropométrica para idosos não institucionalizados no Brasil faz com que os estudos utilizem em suas avaliações padrões internacionais $10,14,15,17,18,19$, o que não é aconselhável, visto as diferenças corporais existentes entre populações 6 .

Com isso, o presente estudo propõe fornecer informações antropométricas e de composição corporal que possam ser utilizadas como padrão de referência de indicadores de gordura e massa muscular de idosos residentes na cidade de Fortaleza.

\section{Material e métodos}

Este estudo é populacional, de base domiciliar, do tipo transversal, com coleta de dados primários. Foram investigados indivíduos com 60 anos e mais, residentes habituais em domicílios particulares em Fortaleza. Foram excluídos do estudo idosos em fase terminal de doença, idosos que apresentassem debilidade clínica grave e os que estivessem ausentes de Fortaleza por mais tempo que a pesquisa de campo naquele setor.

\section{Amostra}

A descrição detalhada do plano de amostragem deste estudo é apresentada no trabalho de Menezes et al. ${ }^{20}$. De forma breve, o cálculo da amostra considerou o número de domicílios de Fortaleza. Para o dimensionamento do número de domicílios a serem visitados foi adotado o modelo de amostragem estratificada proporcional, para o qual se estipulou em $5 \%$ o erro amostral máximo admissível para os testes.

A seleção da amostra foi realizada de acordo com a metodologia utilizada pelo Instituto Brasileiro de Geografia e Estatística (IBGE), que divide as cidades em setores censitários. O plano de amostragem foi elaborado com base em técnicas de processos probabilísticos com estratificação dos bairros e setores censitários de Fortaleza, para garantir a representatividade da área territorial em que a pesquisa foi realizada.

As técnicas amostrais foram planejadas em múltiplas etapas, cuja seleção foi desenvolvida em estágios sucessivos. Inicialmente, foram selecionados, por meio de sorteio, os bairros. Em seguida, foram selecionados aleatoriamente os setores censitários em cada bairro sorteado. A identificação do domicílio foi realizada de forma sistemática. A identificação do primeiro domicílio ocorreu aleatoriamente, considerando como ponto de partida a esquina inicial do setor, em sentido horário. A cada domicílio selecionado, foi saltado um número de domicílios, de acordo com cada setor censitário, e assim sucessivamente, a fim de obter-se melhor distribuição. Caso não residisse idoso na casa selecionada ou o mesmo não aceitasse (recusa) ou não pudesse (perda) participar do estudo, outro idoso foi procurado no domicílio seguinte. No caso de residir mais de um idoso no domicílio, todos foram convidados a participar do estudo.

\section{Coleta dos dados}

A coleta dos dados foi realizada por três equipes de dois entrevistadores cada uma. As equipes que foram a campo para realizar a coleta dos dados eram constituídas por alunos do curso de nutrição da Universidade Estadual do Ceará, os quais foram devidamente treinados pela primeira autora deste estudo.

Os alunos foram treinados considerando os erros de medida aceitáveis. Para as variáveis: perímetro do braço (PB) e DCT foram considerados os valores descritos por Callaway et al. 21 e Harrison et al. ${ }^{22}$, respectivamente. 


\section{Antropometria}

As variáveis antropométricas apresentadas neste estudo são: DCT (mm), AGB $\left(\mathrm{cm}^{2}\right), \mathrm{PB}(\mathrm{cm}), \mathrm{CMB}$ $(\mathrm{cm}), \mathrm{AMB}\left(\mathrm{cm}^{2}\right)$ e $\mathrm{AMBc}\left(\mathrm{cm}^{2}\right)$.

O PB foi aferido de acordo com as técnicas propostas por Callaway et al. 21, utilizando-se fita métrica inelástica, com precisão de $1 \mathrm{~mm}$. A DCT foi aferida utilizando-se o compasso Lange que tem pressão constante de $10 \mathrm{~g} / \mathrm{mm}^{2} 22$.

Com as variáveis DCT e PB foram calculadas as variáveis $\mathrm{AGB}, \mathrm{CMB}, \mathrm{AMB}$ e AMBc. O cálculo da AGB foi realizado a partir da equação citada por Frisancho 5:

$\operatorname{AGB}\left(\mathrm{cm}^{2}\right)=\frac{\left.\mathrm{PB}(\mathrm{cm})^{2}\right]}{4 \pi}-\frac{\mathrm{PB}(\mathrm{cm})-(\pi \times \mathrm{DCT}(\mathrm{cm}))]^{2}}{4 \pi}$

A CMB foi calculada utilizando-se a seguinte equação 22 :

$\mathrm{CMB}(\mathrm{cm})=[\mathrm{PB}(\mathrm{cm})-(\pi \times \mathrm{DCT}(\mathrm{cm}))]$

Para o cálculo da AMB foram utilizadas as equações citadas por Gurney \& Jelliffe 23:

$\operatorname{AMB}\left(\mathrm{cm}^{2}\right): \frac{[\mathrm{PB}(\mathrm{cm})-(\pi \times \mathrm{DCT}(\mathrm{cm}))]^{2}}{4 \pi}$

A estimativa da AMBc foi realizada a partir das equações propostas por Heymsfield et al. 24:

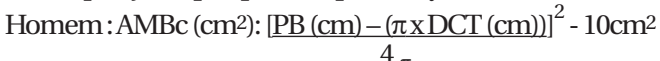
Mulher: $\mathrm{AMBc}\left(\mathrm{cm}^{2}\right)$ : $[\mathrm{PB}(\mathrm{cm})-(\pi \mathrm{xDCT}(\mathrm{cm}))]^{2}-6,5 \mathrm{~cm}^{2}$ $4 \pi$

\section{Análise dos dados}

Os idosos foram agrupados de acordo com o sexo e grupo etário (60-69 anos, 70-79 anos e 80 anos e mais). As variáveis antropométricas são apresentadas sob a forma de média, desvio padrão (DP) e percentil (P5, P10, P25, P50, P75, P90 e P95).

A significância estatística das diferenças de médias entre os sexos foi verificada por meio do teste t-Student. Foram realizadas a análise de variância (ANOVA) e a comparação de Tukey para verificar o efeito do grupo etário nas variáveis antropométricas. Foi utilizado o nível de significância $\alpha=5 \%$. As informações estatísticas foram obtidas por meio do aplicativo estatístico SPSS 10.0 (SPSS Inc., Chicago, Estados Unidos).

\section{Questões éticas}

O presente estudo foi aprovado pelo Comitê de Ética em Pesquisa da Faculdade de Saúde Pública, Universidade de São Paulo, em 7 de agosto de 2001. Os idosos participantes deste estudo assinaram o Termo de Consentimento Livre e Esclarecido, segundo Resolução $n^{o}$. 196, de 10 de outubro de 1996 do Conselho Nacional de Saúde, que regulamenta pesquisas envolvendo seres humanos. No caso dos idosos que por al- gum motivo encontravam-se impossibilitados de assinar o termo de consentimento, foi solicitado ao responsável que o fizesse; caso este não estivesse no domicílio, foi realizada nova visita. Os idosos que se recusaram não participaram do estudo.

\section{Resultados}

Foram selecionados 385 domicílios para comporem a amostra deste estudo, nos quais foram entrevistados 483 idosos (327 mulheres e 156 homens) residentes em Fortaleza. A média etária dos idosos foi de 70,7 anos (DP $=7,8$ ), sendo 70,9 $(\mathrm{DP}=7,9)$ para mulheres e $70,3(\mathrm{DP}=7,8)$ para homens.

Na Tabela 1 são apresentados os valores médios de cada variável antropométrica, segundo sexo e grupo etário. O valor médio da DCT das mulheres foi significativamente superior ao dos homens, assim como os valores da AGB. Os valores médios do $\mathrm{PB}$ foram os mesmos para ambos os sexos. Os valores médios das variáveis $\mathrm{CMB}$, $\mathrm{AMB}$ e $\mathrm{AMBc}$ dos homens são superiores aos das mulheres, cujas diferenças mostraram-se estatisticamente significativas.

A Tabela 1 mostra, ainda, os valores médios das variáveis, de acordo com o grupo etário. Nessa tabela, observa-se tendência do declínio dos valores com o avançar do grupo etário. No caso dos homens, houve diferenças significativas entre as médias do $\mathrm{PB}, \mathrm{AMB}$ e AMBc, com o avançar dos grupos etários. No grupo das mulheres, houve diferenças significativas, com o avançar da idade, para as variáveis DCT e AGB. O teste de comparações múltiplas (teste de Tukey) mostrou que, para a DCT, a diferença somente é estatisticamente significativa entre os grupos de 60-69 anos e 70-79. No caso da AGB, essa diferença foi significativa entre os valores médios dos idosos de 60-69 anos e 70-79, e entre 60-69 e 80 anos e mais. Essa tendência de declínio pode ser observada, mais detalhadamente, na Tabela 2, onde são apresentados os percentis das variáveis antropométricas.

\section{Discussão}

Este trabalho é parte de um estudo mais amplo que constitui a primeira pesquisa populacional, de base domiciliar, realizada em Fortaleza, que envolve coleta de dados primários relativos à antropometria, consumo alimentar e nível sócioeconômico de idosos.

O presente estudo descreve características antropométricas e de composição corporal de 
Médias e desvios padrão (DP) dos indicadores de gordura e massa muscular corporal dos idosos segundo sexo e grupo etário. Fortaleza, Ceará, Brasil.

\begin{tabular}{|c|c|c|c|c|c|c|c|c|}
\hline \multirow[t]{2}{*}{ Variáveis } & \multicolumn{4}{|c|}{ Homem } & \multicolumn{4}{|c|}{ Mulher } \\
\hline & $\mathrm{n}$ & Média & DP & Valor de $\mathrm{p}(\mathrm{F})$ & $\mathrm{n}$ & Média & DP & Valor de $\mathrm{p}(\mathrm{F})$ \\
\hline $\mathrm{DCT}(\mathrm{mm})$ * & & & & $0,888(0,119)$ & & & & $0,000(10,996)$ \\
\hline \multicolumn{9}{|c|}{ Grupo etário (anos) } \\
\hline Total & 156 & 13,0 & 5,2 & & 327 & 21,3 & 7,1 & \\
\hline $60-69$ & 79 & 13,2 & 5,7 & & 159 & 23,0 & 7,3 & \\
\hline $70-79$ & 61 & 12,9 & 4,8 & & 121 & 20,2 & 6,1 & \\
\hline 80 e mais & 16 & 12,6 & 4,3 & & 47 & 18,2 & 7,0 & \\
\hline$A G B\left(\mathrm{~cm}^{2}\right)^{*}$ & & & & $0,383(0,967)$ & & & & $0,000(10,372)$ \\
\hline \multicolumn{9}{|c|}{ Grupo etário (anos) } \\
\hline Total & 156 & 18,1 & 8,0 & & 327 & 28,5 & 12,7 & \\
\hline $60-69$ & 79 & 18,8 & 9,1 & & 159 & 31,6 & 13,9 & \\
\hline $70-79$ & 61 & 17,7 & 6,9 & & 121 & 26,3 & 10,2 & \\
\hline 80 e mais & 16 & 15,9 & 5,4 & & 47 & 23,8 & 11,7 & \\
\hline PB $(\mathrm{cm})$ & & & & $0,010(4,705)$ & & & & $0,001(7,084)$ \\
\hline \multicolumn{9}{|c|}{ Grupo etário (anos) } \\
\hline Total & 156 & 29,3 & 3,2 & & 327 & 29,3 & 4,7 & \\
\hline $60-69$ & 79 & 29,7 & 3,5 & & 159 & 30,2 & 4,9 & \\
\hline $70-79$ & 61 & 29,2 & 2,7 & & 121 & 28,6 & 3,9 & \\
\hline 80 e mais & 16 & 27,1 & 3,1 & & 47 & 27,7 & 4,9 & \\
\hline $\mathrm{CMB}(\mathrm{cm})^{*}$ & & & & $0,002(6,372)$ & & & & $0,083(2,514)$ \\
\hline \multicolumn{9}{|c|}{ Grupo etário (anos) } \\
\hline Total & 156 & 25,2 & 2,6 & & 327 & 22,6 & 3,2 & \\
\hline $60-69$ & 79 & 25,6 & 2,5 & & 159 & 23,0 & 3,4 & \\
\hline $70-79$ & 61 & 25,2 & 2,4 & & 121 & 22,3 & 3,0 & \\
\hline 80 e mais & 16 & 23,1 & 3,0 & & 47 & 22,0 & 3,1 & \\
\hline AMB $\left(\mathrm{cm}^{2}\right)^{*}$ & & & & $0,004(5,699)$ & & & & $0,095(2,375)$ \\
\hline \multicolumn{9}{|c|}{ Grupo etário (anos) } \\
\hline Total & 156 & 50,9 & 10,4 & & 327 & 41,4 & 12,6 & \\
\hline $60-69$ & 79 & 52,6 & 10,5 & & 159 & 42,9 & 14,0 & \\
\hline $70-79$ & 61 & 50,8 & 9,3 & & 121 & 40,2 & 11,0 & \\
\hline 80 e mais & 16 & 43,2 & 11,4 & & 47 & 39,2 & 10,8 & \\
\hline $\mathrm{AMBc}\left(\mathrm{cm}^{2}\right)^{*}$ & & & & $0,004(5,699)$ & & & & $0,095(2,375)$ \\
\hline \multicolumn{9}{|c|}{ Grupo etário (anos) } \\
\hline Total & 156 & 40,9 & 10,4 & & 327 & 34,9 & 12,6 & \\
\hline $60-69$ & 79 & 42,6 & 10,5 & & 159 & 36,4 & 14,0 & \\
\hline $70-79$ & 61 & 40,8 & 9,3 & & 121 & 33,7 & 10,9 & \\
\hline 80 e mais & 16 & 33,2 & 11,4 & & 47 & 32,7 & 10,8 & \\
\hline
\end{tabular}

DCT: dobra cutânea tricipital; AGB: área de gordura do braço; PB: perímetro do braço; CMB: circunferência muscular do braço; AMB: área muscular do braço; AMBc: área muscular do braço corrigida.

* Diferença estatisticamente significativa dos valores médios entre os sexos.

uma amostra representativa dos idosos de Fortaleza, cujos resultados obtidos podem ser extrapoladas para a população idosa do município.

As variáveis antropométricas descritas neste estudo são comumente utilizadas em estudos antropométricos, sejam eles populacionais 1,3,9,11,12,25 ou em grupos específicos da população idosa 2,13 .
De acordo com as variáveis antropométricas, observa-se que a média da DCT das mulheres foi significativamente superior à dos homens. No Brasil, Marucci \& Barbosa 10, em estudo populacional com idosos residentes em São Paulo, encontraram média de DCT das mulheres $(25,22 \mathrm{~mm})$ superior à dos homens $(13,71 \mathrm{~mm})$, cuja diferença mostrou-se estatisticamente significativa. A média dos homens foi similar a deste 
Percentis dos indicadores de gordura e massa muscular corporal dos idosos segundo sexo e grupo etário. Fortaleza, Ceará, Brasil.

\begin{tabular}{|c|c|c|c|c|c|c|c|c|}
\hline \multirow[t]{2}{*}{ Variáveis antropométricas } & \multirow[t]{2}{*}{$\mathrm{n}$} & \multicolumn{7}{|c|}{ Percentil } \\
\hline & & 5 & 10 & 25 & 50 & 75 & 90 & 95 \\
\hline \multicolumn{9}{|l|}{$\mathrm{DCT}(\mathrm{mm})$} \\
\hline Mulher & 327 & 10,1 & 12,0 & 16,2 & 21,0 & 25,8 & 31,0 & 33,8 \\
\hline \multicolumn{9}{|l|}{ Grupo etário (anos) } \\
\hline $60-69$ & 159 & 11,5 & 12,7 & 17,2 & 22,7 & 28,0 & 32,3 & 35,0 \\
\hline $70-79$ & 121 & 9,9 & 12,0 & 16,4 & 20,0 & 23,4 & 29,4 & 31,5 \\
\hline 80 e mais & 47 & 6,3 & 8,5 & 12,7 & 19,2 & 23,3 & 27,5 & 30,1 \\
\hline Homem & 156 & 5,4 & 6,8 & 8,8 & 12,5 & 15,7 & 20,8 & 22,7 \\
\hline \multicolumn{9}{|l|}{ Grupo etário (anos) } \\
\hline $60-69$ & 79 & 4,8 & 6,0 & 9,0 & 12,8 & 15,7 & 22,2 & 24,2 \\
\hline $70-79$ & 61 & 6,7 & 7,1 & 8,5 & 12,2 & 15,9 & 20,0 & 21,8 \\
\hline 80 e mais & 16 & 6,0 & 6,2 & 9,4 & 12,6 & 15,3 & 19,6 & 20,5 \\
\hline \multicolumn{9}{|l|}{$\mathrm{AGB}\left(\mathrm{cm}^{2}\right)$} \\
\hline Mulher & 327 & 10,6 & 13,6 & 20,0 & 27,0 & 35,1 & 45,9 & 52,0 \\
\hline \multicolumn{9}{|l|}{ Grupo etário (anos) } \\
\hline $60-69$ & 159 & 13,4 & 14,6 & 21,4 & 29,3 & 39,5 & 51,4 & 57,0 \\
\hline $70-79$ & 121 & 10,3 & 13,9 & 19,2 & 25,7 & 30,2 & 39,3 & 48,3 \\
\hline 80 e mais & 47 & 5,5 & 8,8 & 13,8 & 23,7 & 32,1 & 40,1 & 47,4 \\
\hline Homem & 156 & 6,3 & 8,6 & 11,1 & 17,7 & 22,4 & 29,7 & 32,7 \\
\hline \multicolumn{9}{|l|}{ Grupo etário (anos) } \\
\hline $60-69$ & 79 & 5,2 & 7,0 & 10,7 & 18,0 & 23,8 & 32,1 & 35,2 \\
\hline $70-79$ & 61 & 8,7 & 9,2 & 11,1 & 16,9 & 22,2 & 28,0 & 31,0 \\
\hline 80 e mais & 16 & 5,9 & 7,4 & 13,4 & 15,9 & 19,4 & 23,4 & 26,7 \\
\hline \multicolumn{9}{|l|}{ PB (cm) } \\
\hline Mulher & 327 & 22,1 & 24,0 & 26,0 & 29,1 & 32,0 & 35,2 & 37,3 \\
\hline \multicolumn{9}{|l|}{ Grupo etário (anos) } \\
\hline $60-69$ & 159 & 23,8 & 24,5 & 26,8 & 29,6 & 33,1 & 36,2 & 39,3 \\
\hline $70-79$ & 121 & 22,1 & 23,7 & 26,0 & 28,4 & 30,8 & 33,6 & 35,9 \\
\hline 80 e mais & 47 & 18,3 & 21,3 & 24,0 & 28,4 & 31,3 & 34,0 & 35,5 \\
\hline Homem & 156 & 24,1 & 25,4 & 27,3 & 29,1 & 31,5 & 33,0 & 34,2 \\
\hline \multicolumn{9}{|l|}{ Grupo etário (anos) } \\
\hline $60-69$ & 79 & 24,1 & 25,4 & 27,6 & 29,8 & 31,6 & 34,1 & 36,0 \\
\hline $70-79$ & 61 & 24,6 & 26,0 & 27,5 & 29,0 & 31,6 & 32,8 & 33,0 \\
\hline 80 e mais & 16 & 20,5 & 22,2 & 25,4 & 26,7 & 29,2 & 31,8 & 31,9 \\
\hline \multicolumn{9}{|l|}{$\mathrm{CMB}(\mathrm{cm})$} \\
\hline Mulher & 327 & 18,2 & 18,9 & 20,3 & 22,5 & 24,3 & 26,4 & 28,3 \\
\hline \multicolumn{9}{|l|}{ Grupo etário (anos) } \\
\hline $60-69$ & 159 & 18,8 & 19,4 & 20,7 & 22,6 & 24,4 & 26,8 & 29,1 \\
\hline $70-79$ & 121 & 17,8 & 18,8 & 20,1 & 22,1 & 24,3 & 25,9 & 28,1 \\
\hline 80 e mais & 47 & 16,2 & 18,2 & 19,8 & 21,6 & 24,5 & 26,1 & 27,0 \\
\hline Homem & 156 & 20,5 & 21,6 & 23,7 & 25,3 & 26,7 & 28,4 & 29,3 \\
\hline \multicolumn{9}{|l|}{ Grupo etário (anos) } \\
\hline $60-69$ & 79 & 21,2 & 22,4 & 24,0 & 25,8 & 27,0 & 28,8 & 29,7 \\
\hline $70-79$ & 61 & 20,4 & 22,1 & 23,8 & 25,2 & 26,5 & 28,4 & 28,7 \\
\hline 80 e mais & 16 & 18,7 & 19,5 & 20,6 & 22,9 & 25,4 & 28,3 & 29,1 \\
\hline \multicolumn{9}{|l|}{$\mathrm{AMB}\left(\mathrm{cm}^{2}\right)$} \\
\hline Mulher & 327 & 26,4 & 28,5 & 32,8 & 40,1 & 47,2 & 55,6 & 63,7 \\
\hline \multicolumn{9}{|l|}{ Grupo etário (anos) } \\
\hline $60-69$ & 159 & 28,2 & 29,9 & 34,1 & 40,6 & 47,3 & 57,0 & 67,2 \\
\hline $70-79$ & 121 & 25,2 & 28,0 & 32,2 & 38,8 & 47,1 & 53,3 & 62,9 \\
\hline 80 e mais & 47 & 21,0 & 26,3 & 31,1 & 37,1 & 47,8 & 54,4 & 58,1 \\
\hline
\end{tabular}

(continua) 
Tabela 2 (continuação)

\begin{tabular}{|c|c|c|c|c|c|c|c|c|}
\hline \multirow[t]{2}{*}{ Variáveis antropométricas } & \multirow[t]{2}{*}{$\mathrm{n}$} & \multicolumn{7}{|c|}{ Percentil } \\
\hline & & 5 & 10 & 25 & 50 & 75 & 90 & 95 \\
\hline Homem & 156 & 33,4 & 37,0 & 44,6 & 51,0 & 56,9 & 64,3 & 68,5 \\
\hline \multicolumn{9}{|l|}{ Grupo etário (anos) } \\
\hline $60-69$ & 79 & 35,8 & 40,0 & 45,7 & 52,8 & 58,1 & 65,9 & 70,0 \\
\hline $70-79$ & 61 & 33,3 & 38,7 & 45,2 & 50,7 & 56,0 & 64,1 & 65,6 \\
\hline 80 e mais & 16 & 27,7 & 30,2 & 33,7 & 41,7 & 51,4 & 63,6 & 67,2 \\
\hline \multicolumn{9}{|l|}{$\mathrm{AMBc}\left(\mathrm{cm}^{2}\right)$} \\
\hline Mulher & 327 & 19,9 & 22,0 & 26,3 & 33,6 & 40,7 & 49,1 & 57,2 \\
\hline \multicolumn{9}{|l|}{ Grupo etário (anos) } \\
\hline $60-69$ & 159 & 21,7 & 23,4 & 27,6 & 34,1 & 40,8 & 50,5 & 60,7 \\
\hline $70-79$ & 121 & 18,7 & 21,5 & 25,7 & 32,3 & 40,6 & 46,8 & 56,4 \\
\hline 80 e mais & 47 & 14,5 & 19,8 & 24,6 & 30,6 & 41,3 & 47,9 & 51,6 \\
\hline Homem & 156 & 23,4 & 27,0 & 34,6 & 41,0 & 46,9 & 54,3 & 58,5 \\
\hline \multicolumn{9}{|l|}{ Grupo etário (anos) } \\
\hline $60-69$ & 79 & 25,8 & 30,0 & 35,7 & 42,8 & 48,1 & 55,9 & 60,0 \\
\hline $70-79$ & 61 & 23,3 & 28,7 & 35,2 & 40,7 & 46,0 & 54,1 & 55,6 \\
\hline 80 e mais & 16 & 17,7 & 20,2 & 23,7 & 31,7 & 41,4 & 53,6 & 57,2 \\
\hline
\end{tabular}

DCT: dobra cutânea tricipital; AGB: área de gordura do braço; PB: perímetro do braço; CMB: circunferência muscular do braço; AMB: área muscular do braço; $\mathrm{AMBc}$ : área muscular do braço corrigida.

estudo, no entanto a das mulheres foi superior. Mastroeni 19, em pesquisa com idosos em Joinville, Santa Catarina, Brasil, encontrou média entre as mulheres $(21,9 \mathrm{~mm})$ similar a deste estudo e dos homens, inferior $(10,7 \mathrm{~mm})$.

Diferenças nas médias da DCT entre os grupos etários indicam um possível efeito da idade na alteração da variável, principalmente das mulheres, a qual mostrou-se estatisticamente significativa. A média da DCT das mulheres de 60-69 anos foi $12,2 \%$ maior que das mulheres de $70-79$ anos e $20,9 \%$ maior que das mulheres de 80 anos e mais. Essa tendência relacionada ao grupo etário também pode ser observada na distribuição em percentil. Os homens apresentaram discreta diminuição nos valores de DCT, sendo que ao verificar os dados do percentil 50, observa-se que a DCT aumentou do grupo etário 60-69 anos para o de 70-79, a qual elevou entre os idosos com 80 anos e mais.

Achados semelhantes foram observados por outros autores, tanto em estudos que apresentam seus dados em percentil 4,8,12,13, quanto em estudos que apresentam seus dados sob a forma de médias ${ }^{7}$. Santos et al. 11 observaram aumento das médias de DCT entre as mulheres de 60-64 anos e 65-69, as quais apresentaram diminuição no avançar de todos os outros grupos etários. Entretanto, há que se considerar que Santos et al. 11 trabalharam com intervalos de grupos etários mais curtos, o que pode justificar esse aumento em percentil, visto que, apesar desse aumento, os autores afirmam que a DCT diminuiu mais rapidamente com o avançar da idade entre as mulheres que entre os homens. Observa-se, então, que, assim como o sexo, a idade é um fator determinante na alteração dos valores da variável. Tendência de diminuição dos valores de DCT com o avançar da idade observada neste estudo foi também encontrada por outros autores 1,8,9,11,12,13.

Como visto, apesar de os valores médios serem diferentes, os resultados deste estudo corroboram achados de estudos realizados no Brasil, assim como em outros países, os quais indicam maior acúmulo de gordura subcutânea nas mulheres, quando comparadas aos homens $7,8,9,10,11,19$.

A AGB, assim como a DCT, é uma variável usada como indicadora de gordura e também utilizada em estudos antropométricos com idosos 5,26,27. Os valores médios da AGB dos idosos deste estudo, os quais são maiores entre as mulheres, mostram maior reserva de gordura quando comparados aos homens. Ao comparar os resultados deste estudo ao de Zverev \& Chisi 27 , realizado com indivíduos da área rural da Malásia, observa-se valores similares, em que as mulheres apresentam valores médios superiores aos homens, cujas diferenças entre os sexos mostram-se significativas. Resultados semelhantes foram observados por Frisancho 5.

$\mathrm{O}$ valor médio do $\mathrm{PB}$ de homens e mulheres foi o mesmo $(29,3 \mathrm{~cm})$. Com relação aos grupos 
etários, tanto homens como mulheres apresentaram diminuição nos valores médios com o avançar da idade. Sendo que, entre as mulheres, essa diminuição mostrou-se mais pronunciada. Assim como neste trabalho, Santos et al. 11, em estudo populacional conduzido com idosos no Chile, observaram que os valores médios do $\mathrm{PB}$ diminuíram com o avançar do grupo etário, tanto em homens quanto em mulheres. Kuczmarski et al. 9, em estudo populacional com idosos avaliados na NHANES III, cujos dados são apresentados em percentil, verificaram diminuição dos valores de PB no percentil 50, em ambos os sexos, com o avançar do grupo etário. Como visto, apesar de apresentarem valores diferentes, a tendência de diminuição do PB é confirmada por outros estudos. A diminuição do PB com o avançar dos grupos etários, verificada tanto nos valores médios quanto em percentil, pode ser devida à perda de massa adiposa e muscular, característica do processo de envelhecimento, que, por conseguinte, ocasiona alteração nesta variável. Achados semelhantes são comumente registrados por estudos com idosos 2,4,6,7,11,12,13,27.

Apesar de o PB ser uma variável antropométrica que reflete reserva muscular e de gordura 24,28,29,30, a mesma é também indicada para verificar reserva de massa muscular; no entanto, sua utilização como medida independente não é aconselhável por representar o somatório das áreas constituídas pelos tecidos ósseo, muscular, gorduroso e epitelial do braço 21,31.

As variáveis indicativas de reserva de massa muscular (CMB, AMB e AMBc), apresentaram valores médios maiores entre os homens, quando comparados às das mulheres, cujas diferenças mostraram-se estatisticamente significativas. Os valores médios dessas variáveis apresentaram diminuição com o avançar do grupo etário, no entanto apenas entre os homens estas diferenças foram estatisticamente significativas. Tendência de diminuição também pode ser observada nos dados em percentil. Estudos antropométricos utilizando indicadores de massa muscular, assim como neste estudo, têm observado maior depósito de massa muscular entre os homens, assim como sua diminuição com o avanço do grupo etário, que ocorre tanto em homens como em mulheres; no entanto, de forma mais pronunciada entre os homens 9,26,32.
Essa diminuição nos valores das variáveis indicativas de massa muscular, de acordo com o grupo etário, é preocupante, uma vez que, alterações musculares levam a manifestações clínicas que podem influenciar de forma negativa na vida do idoso, visto que a perda de massa corporal tem impacto sobre sua capacidade funcional 33 . A força muscular, diretamente determinada pela quantidade de massa muscular, é uma medida preditora de independência e mobilidade em idosos 34. Dessa forma, medidas indicadoras de massa muscular podem ser utilizadas em programas de avaliação nutricional, auxiliando na detecção de riscos, de forma a garantir intervenções adequadas, melhorando a qualidade de vida do idoso.

Como visto, os resultados deste estudo mostram que os padrões das mudanças corporais são semelhantes, no entanto a magnitude das diferenças varia entre os grupos estudados, possivelmente pela conhecida diferença corporal existente entre as populações. Estudo realizado por Delarue et al. 6 observou diferenças corporais significativas entre idosos de duas localidades na França. Além das diferenças entre populações, há ainda, alguns fatores que limitam a utilização de padrões internacionais, como: características metodológicas, seleção da amostra e critérios de inclusão e exclusão. Outro fator que pode ser limitante para a avaliação e que deve ser considerado é a forma de apresentação dos dados (média ou percentil, categorização dos grupos etários - 5 em 5 ou 10 em 10 anos).

Alguns autores, diante da necessidade de padrões de referência locais, sugerem que os resultados de seus estudos, populacionais ou não, sejam utilizados com essa finalidade 5,6,7,9,12,13,25.

Com isso, confirma-se a necessidade de estudos antropométricos em amostra representativa da população idosa, que permitam a construção de padrões de referência locais, estratificados por sexo e grupo etário, pois não é recomendável que variáveis de idosos sejam comparadas com as de outros grupos etários devido às alterações corporais que acompanham o processo de envelhecimento; assim como, não é aconselhável a utilização de padrões de referência internacionais.

Dessa forma, sugere-se que as informações obtidas neste estudo sejam utilizadas como padrão de referência para indicadores de gordura e massa muscular de idosos residentes na cidade de Fortaleza. 


\section{Resumo}

O objetivo deste estudo foi descrever as variáveis indicadoras de gordura e massa muscular dos idosos de Fortaleza, Ceará, Brasil. Este estudo é populacional, transversal, domiciliar, realizado com idosos (60 anos ou mais) residentes em Fortaleza. As variáveis verificadas foram: dobra cutânea tricipital (DCT), área de gordura do braço (AGB), perímetro do braço (PB), circunferência muscular do braço (CMB), área muscular do braço (AMB) e área muscular do braço corrigida $(A M B c)$. Foram avaliados 483 idosos. O valor médio da DCT das mulheres foi significativamente superior ao dos homens ( $p<0,05)$, assim como o valor médio da $A G B(p<0,05)$. As variáveis $C M B, A M B$ e AMBc apresentaram valor médio maior entre os homens, quando comparados às mulheres $(p<0,05)$. Em todas as variáveis houve diminuição dos valores médios e percentil com o avançar dos grupos etários, com exceção do percentil da DCT para as mulheres. O padrão antropométrico e de composição corporal dos idosos deste trabalho segue a tendência de outros estudos, no entanto, os valores diferem. Com isso, sugere-se que as informações deste estudo sejam utilizadas como padrão de referência para idosos de Fortaleza.

Antropometria; Composição Corporal; Idoso

\section{Referências}

1. Forster S, Gariballa S. Age as a determinant of nutritional status: a cross sectional study. Nutr J 2005; 4:28-32.

2. Sánchez-García S, García-Peña C, Duque-López MX, Juárez-Cedillo T, Cortés-Núñez AR, ReyesLópes S. Anthropometric measures and nutritional status in a healthy elderly population. BMC Public Health 2007; 7:2-10.

3. Frisancho AR. New norms of upper limb fat and muscle areas for assessment of nutritional status. Am J Clin Nutr 1981; 34:2540-5.

4. Falciglia G, O'Connor J, Gedling E. Upper arm anthropometric norms in elderly white subjects. J Am Diet Assoc 1988; 88:569-74.

5. Frisancho AR. Anthropometric standards for the assessment of growth and nutritional status. Ann Arbor: University of Michigan Press; 1990.

6. Delarue J, Constans T, Malvy D, Pradignac A, Couet C, Lamisse F. Anthropometric values in an elderly French population. Br J Nutr 1994; 71:295-302.

\section{Colaboradores}

T. N. Menezes participou na elaboração e desenvolvimento do projeto, recrutamento e treinamento dos entrevistadores, digitação, análise e discussão dos dados, redação do artigo. M. F. N. Marucci contribuiu na orientação da pesquisa, análise e discussão dos dados e revisão final do artigo.

\section{Agradecimentos}

Secretaria de Saúde do Estado do Ceará (SESA) em convênio com o Centro de Treinamento e Desenvolvimento (CETREDE) (Processo no. 02429195-1) e Conselho Nacional de Desenvolvimento Científico e Tecnológico (CNPq), pelo apoio financeiro.
7. Velázquez-Alva MC, Castillo-Martínez L, IrigoyenCamacho E, Zepeda-Zepeda MA, Gutiérrez-Robledo LM, Cisneros-Moysen P. Estudio antropométrico en un grupo de hombres y mujeres de la tercera edad en la Ciudad de México. Salud Pública Méx 1996; 38:466-74

8. Suriah AR, Zalifah MK, Zainorni MJ, Shafawi S, Mimie Suraya S, Zarina N, et al. Anthropometric measurements of the elderly. Malaysian Journal of Nutrition1998; 4:55-63.

9. Kuczmarski MF, Kuczmarski RJ, Najjar M. Descriptive anthropometric reference data for older Americans. J Am Diet Assoc 2000; 100:59-66.

10. Marucci MFN, Barbosa AR. Estado nutricional e capacidade física. In: Lebrão ML, Duarte YAO, organizadores. SABE - Saúde, Bem-estar e Envelhecimento. O projeto SABE no Município de São Paulo: uma abordagem inicial. 1a Ed. Brasília: Organização Pan-Americana da Saúde; 2003. p. 95117. 
11. Santos JL, Albala C, Lera L, García C, Arroyo P, Pérez-Bravo F, et al. Anthropometric measurements in the elderly population of Santiago, Chile. Nutrition 2004; 20:452-7.

12. Barbosa AR, Souza JMP, Lebrão ML, Laurenti $R$, Marucci MFN. Anthropometry of elderly residents in the city of São Paulo, Brazil. Cad Saúde Pública 2005; 21:1929-38.

13. Menezes TN, Marucci MFN. Antropometria de idosos residentes em instituições geriátricas, Fortaleza, CE. Rev Saúde Pública 2005; 39:169-75.

14. Tavares EL, Anjos LA. Perfil antropométrico da população idosa brasileira. Resultados da Pesquisa Nacional sobre Saúde e Nutrição. Cad Saúde Pública 1999; 15:759-68.

15. Barreto SM, Passos VMA, Lima-Costa MFF. Obesity and underweight among Brazilian elderly: the Bambuí Health and Aging Study. Cad Saúde Pública 2003; 19:605-12.

16. Gray GE, Gray LK. Validity of anthropometric norms used in the assessment of hospitalized patients. JPNE J Parenter Enteral Nutr 1979; 3:366-8.

17. Menezes TN. Avaliação antropométrica e do consumo alimentar de idosos residentes em instituições geriátricas da cidade de Fortaleza/Ceará [Dissertação de Mestrado]. São Paulo: Faculdade de Saúde Pública, Universidade de São Paulo; 2000.

18. Barbosa AR. Estado nutricional e sua associação com força muscular, flexibilidade e equilíbrio de idosos residentes no Município de São Paulo [Tese de Doutorado]. São Paulo: Faculdade de Ciências Farmacêuticas/Faculdade de Economia, Administração e Contabilidade/Faculdade de Saúde Pública, Universidade de São Paulo; 2004.

19. Mastroeni MF. Estado nutricional e consumo de macronutrientes de idosos da cidade de Joinville, SC [Tese de Doutorado]. São Paulo: Faculdade de Saúde Pública, Universidade de São Paulo; 2004.

20. Menezes TN, Lopes FJM, Marucci MFN. Estudo domiciliar da população idosa de Fortaleza/CE: aspectos metodológicos e características sócio-demográficas. Rev Bras Epidemiol 2007; 10:168-71.

21. Callaway CW, Chumlea WC, Bouchard C, Himes JH, Lohman TG, Martin AD, et al. Circumferences. In: Lohman TG, Roche AF, Martorell R, editors. Anthropometric standardization reference manual. Champaign: Human Kinetics Books; 1988. p. 3954.

22. Harrison GG, Buskirk ER, Carter JEL, Johnston FE, Lohman TG, Pollock ML, et al. Skinfold thicknesses and measurement technique. In: Lohman TG, Roche AF, Martorell R, editors. Anthropometric standardization reference manual. Champaign: Human Kinetics Books; 1988. p. 55-70.
23. Gurney JM, Jelliffe DB. Arm anthropometry in nutritional assessment: nomogram for rapid calculation of muscle circumference and cross-sectional muscle and fat areas. Am J Clin Nutr 1973; 26:9125.

24. Heymsfield SB, McManus C, Smith J, Stevens V, Nixon DW. Anthropometric measurement of muscle mass: revised equations for calculating bonefree arm muscle area. Am J Clin Nutr 1982; 36:68090.

25. Perissinotto E, Pisent C, Sergi G, Grigoletto F, Enzi G. Anthropometric measurements in the elderly: age and gender differences. Br J Nutr 2002; 87:17786.

26. Corish CA, Kennedy NP. Anthropometric measurements from a cross-sectional survey of Irish free-living elderly subjects with smoothed centile curves. Br J Nutr 2003; 89:137-45.

27. Zverev Y, Chisi J. Anthropometric indices in rural Malawians aged 45-75 years. Ann Hum Biol 2004; 31:29-37.

28. Bartali B, Benvenuti E, Corsi AM, Bandinelli S, Russo CR, Di Lorio A, et al. Changes in anthropometric measures in men and women across the life-span: findings from the InCHIANTI study. Soz Praventivmed 2002; 47:336-48.

29. Frisoni GB, Franzoni S, Rozzini R, Ferrucci L, Boffelli S, Trabucchi M. A nutritional index predicting mortality in the nursing home. J Am Geriatr Soc $1994 ; 42: 1167-72$.

30. Miller MD, Crotty M, Giles LC, Bannerman E, Whitehead C, Cobiac L, et al. Corrected arm muscle area: an independent predictor of long-term mortality in community-dwelling older adults? J Am Geriatr Soc 2002; 50:1272-7.

31. Frisancho AR. Triceps skinfold and upper arm muscle size norms for assessment of nutritional status. Am J Clin Nutr 1974; 27:1052-7.

32. Bishop CW, Bowen PE, Ritchey SJ. Norms for nutritional assessment of American adults by upper arm anthropometry. Am J Clin Nutr 1981; 34: 25309.

33. Chumlea WC, Guo SS, Glasser RM, Vellas BJ. Sarcopenia, function and health. J Nutr Health Aging 1997; 1:7-12.

34. Fiatarone MA, Marks EC, Ryan ND, Meredith N, Lipsitz LA, Evans WJ. High-intensity strength training in nonagenarians: effects on skeletal muscle. JAMA 1980; 263:3029-34.

Recebido em 13/Jun/2006

Versão final reapresentada em 16/Mar/2007

Aprovado em 23/Mai/2007 